APS

physics

This is the accepted manuscript made available via CHORUS. The article has been published as:

\title{
Free Surface Relaxations of Star-Shaped Polymer Films
} Emmanouil Glynos, Kyle J. Johnson, Bradley Frieberg, Alexandros Chremos, Suresh Narayanan, Georgios Sakellariou, and Peter F. Green Phys. Rev. Lett. 119, 227801 - Published 28 November 2017 DOI: 10.1103/PhysRevLett.119.227801 


\section{Free Surface Relaxations of Star-Shaped Polymer Films}

Emmanouil Glynos, ${ }^{1, *}$ Kyle J. Johnson, ${ }^{2,3}$ Bradley Frieberg, ${ }^{3,4,+}$ Alexandros Chremos ${ }^{5}$, Suresh Narayanan, ${ }^{7}$ Georgios Sakellariou, ${ }^{8}$ and Peter F. Green, ${ }^{2,3,4,5}$ *

${ }^{1}$ Institute of Electronic Structure and Laser, Foundation for Research and Technology-Hellas, P.O. Box 1385, 71110 Heraklion, Crete, Greece

${ }^{2}$ Department of Material Science and Engineering, ${ }^{3}$ Biointeraces Institute, ${ }^{4}$ Macromolecular Science and Engineering, University of Michigan, Ann Arbor, Michigan 48109, USA

${ }^{5}$ National Renewable Energy Laboratory (NREL), Golden CO, 80401

${ }^{6}$ Materials Science and Engineering Division, National Institute of Standards and Technology, Gaithersburg, MD, 20899, USA

${ }^{7}$ X-ray Science Division, Argonne National Laboratory, Argonne, Illinois 60439, USA

${ }^{8}$ Department of Chemistry, National and Kapodistrian University of Athens, Panepistimiopolis, Zografou, 15771, Athens Greece 


\begin{abstract}
The surface relaxation dynamics of supported star-shaped polymer thin films are shown to be slower than the bulk, persisting up to temperatures at least 50 degrees above the bulk glass transition temperature $T_{\mathrm{g}}^{\text {bulk }}$. This behavior, exhibited by star-shaped polystyrenes (SPSs) with functionality $f=8$-arms and molecular weights per arm $M_{\mathrm{arm}}<M_{\mathrm{e}}\left(M_{\mathrm{e}}\right.$ is the entanglement molecular weight), is shown by molecular dynamics simulations to be associated with a preferential localization of these macromolecules at the free surface. This new phenomenon is in notable contrast to that of linear chain polymer thin film systems where the surface relaxations are enhanced in relation to the bulk; this enhancement persists only for a limited temperature range above the bulk $T_{\mathrm{g}}^{\text {bulk }}$. Evidence of the slow surface dynamics, compared to the bulk, for temperatures well above $T_{\mathrm{g}}$ and at length and time scales not associated with the glass transition has not previously been reported for polymers.
\end{abstract}


The manner in which dynamics at the free surfaces of materials differ from the bulk is of fundamental and practical interest, affecting physical phenomena such as wetting, adhesion and tribology. In polymers this behavior occurs over various time and length-scales and attracts considerable attention, theoretically, experimentally and computationally [1-5]. In linear-chain polymer systems, not unlike metals or semiconductors, the surface dynamics are generally faster than the bulk. Below the bulk glass transition temperature $T_{\mathrm{g}}^{\text {bulk }}$ of linear-chain polymers, experiments reveal the existence of a liquid-like layer, a few nanometers thick, at the free surface [5-7]. The enhanced configurational freedom of chain segments at the free interface is responsible this enhanced surface dynamics, and an associated lower free surface glass transition temperature $T_{\mathrm{g}}^{\text {surf }}$.

This free surface layer of enhanced segmental dynamics persists for a relatively "narrow" temperature window above the bulk $T_{\mathrm{g}}[8-10]$. The very first XPCS measurements of supported entangled linear polystyrene (PS) polymer films showed that the free surface viscosities, $\eta_{\mathrm{XPCS}}$, at temperatures $T>T_{\mathrm{g}}^{\text {bulk }}+30 \mathrm{~K}$, are equal to their bulk zero shear viscosities, $\eta_{0}$. The surface relaxation times were described by the hydrodynamic continuum theory (HCT) that assumes non-slip boundary conditions at the liquid/substrate interface and that the film possesses a uniform viscosity $\eta[11,12]$. It predicts that the relaxation time is proportional to $\eta / \gamma$, where $\gamma$ is the surface tension of the film [13]. This finding is consistent with the notion that for temperatures sufficiently high above $T_{\mathrm{g}}$, the surface and bulk dynamics should be similar.[9] Only within a limited temperature range above $T_{\mathrm{g}}^{\text {bulk }}$, i.e. at $T_{\mathrm{g}}<T<T_{\mathrm{g}}+20 \mathrm{~K}$, are the surface dynamics faster than the bulk as faster segmental dynamics are manifested within the time (1$\left.10^{3} \mathrm{sec}\right)$ and length scales $\left(10-10^{3} \mathrm{~nm}\right)$ of the XPCS measurements and the surface relaxation times were shorter than that of the corresponding bulk zero shear viscosity [14]. 
The relaxation dynamics of star-shaped polymers, and branched polymers in general, differ significantly from linear chain polymers; linear -chain polymers are known to diffuse via slithering motions -Rouse dynamics for unentangled linear chains and reptation, involving the curvilinear motions of long chains along "tubes," and tube-renewal processes, for entangled molecules. For entangled star-shaped molecules, mechanisms that require the retraction of an "arm" of the molecule are essential in order to facilitate viscous flow. This facilitation process dictates that segmental relaxations would occur hierarchically -segments relax rapidly near the free ends of the chains and slower, with limited excursions, closer to the branch points of each molecule. The dynamics of branched polymers are therefore characterized by a wider distribution of relaxation times and length-scales [15]. Foster and co-workers, recently investigated the effect macromolecular architecture on the dynamics of polymer films.[16-18] In contrast to linear-chain and cyclic molecules [16], branching was shown to have a significant effect on the surface fluctuation dynamics $[17,18]$. Specifically with the use of XPCS they showed that for unentangled 6-arm star-shaped and six-end end branched stars, the surface viscosities were higher than the bulk viscosities. For molecules characterized by extreme branching (6-pom and bottle brushes) the surface and bulk viscosities were more comparable [17].

Recently, we showed that for 8-arm star polymer films with a molecular weight per arm of $M_{\mathrm{w}}^{\text {arm }}=10 \mathrm{~kg} / \mathrm{mol} . T_{\mathrm{g}}^{\text {surf }}>T_{\mathrm{g}}^{\text {bulk }} \cdot[6,19,20]$ However $T_{\mathrm{g}}^{\text {surf }}$ became gradually lower than $T_{\mathrm{g}}^{\text {bulk }}$ as $M_{\mathrm{w}}$ arm became much larger than $M_{\mathrm{e}}$, the molecular weight between entanglements; this trend is consistent with the behavior of linear chain polymers $[6,19,20]$. Molecular dynamics simulations revealed that this behavior $-T_{\mathrm{g}}^{\text {surf }}>T_{\mathrm{g}}^{\text {bulk }}$-might be due to positional correlations of the starshaped molecular at the free surface of the film [20]. In this letter we demonstrate using XPCS 
that the in contrast to the linear chain polymers, the free surface layer relaxations are slow compared to the bulk and this persists to temperatures $T>T_{\mathrm{g}}+50 \mathrm{~K}$. Molecular dynamics simulations of supported thin polymer films indicate that the slow dynamics, and $T_{\mathrm{g}}^{\text {surf }}>T_{\mathrm{g}}^{\text {bulk }}$, would be due to a preferential localization of these molecules at the free surface. The XPCS measurements indicate that the dynamics are restricted spatially, or "caged," at temperatures tens of degrees above the glass transition temperature, due to the persistence of structural ordering. The slow surface dynamics compared to the bulk at length and time-scales, not associated with the glass transition, have not been shown before, to the best of our knowledge.

Perhaps the best experimental insights into the surface dynamics of polymer films may be obtained using XPCS. The surface fluctuation dynamics were measured using XPCS, characterized by the normalized intensity-intensity autocorrelation function of the coherent surface scattering $g_{2}(q, t)$, given by $g_{2}\left(q_{\|}, t\right)=\left\langle I\left(q_{\|}, t^{\prime}\right) I\left(q_{\|}, t^{\prime}+t\right)\right\rangle /\left\langle I\left(q_{\|}, t^{\prime}\right)\right\rangle^{2}$, where $I\left(q_{\|}, t^{\prime}\right)$ is the scattering intensity for the in-plane vector at time $t^{\prime}$, and $t$ is the delay time. For highly viscous polymers, the capillary waves are over damped so $g_{2}(q, t)$ may be described by an exponential decay: $g_{2}(q, t)=1+\beta \exp \left[-(2 t / \tau)^{a}\right]$, where $\beta$ is the speckle contrast, $\alpha(0<\alpha<1)$ is a stretching exponent, and $\tau=\tau\left(q_{\|}\right)$is the average relaxation time; where $\alpha=1$ corresponds to a simple exponential decay.

Films of various polymers, with thickness of approximately $h=200 \mathrm{~nm}$, were examined using XPCS. Details of the experimental conditions and the analysis of the experimental data are reported in the Supplemental information [21]. Fig. S1 shows raw $g_{2}$ data for linear PS films with $M_{\mathrm{w}}=13 \mathrm{~kg} / \mathrm{mol}$. (LPS-13K) and for 8-arm stars with $M_{\mathrm{w}}^{\text {arm }}=57 \mathrm{~kg} / \mathrm{mol}$. (SPS-8-57K) for a temperature $40 \mathrm{~K}$ higher than the corresponding bulk $T_{\mathrm{g}}$. For the LPS- $13 \mathrm{~K}$, the $g_{2}$ data were 
described well by a simple exponential decay (in accordance with literature [22]), whereas for SPS-8-57K, $g_{2}$ followed a stretched exponential decay.

The XPCS data suggest surprising differences between surface relaxation processes that occur in films composed of linear chains and of SPS molecules of different arm lengths. First, our studies confirm that the surface dynamics measured by XPCS on linear chain PS films are in excellent agreement with the values of the zero shear viscosities, measured using bulk rheology; the XPCS relaxation times can be described by HCT (Fig. 1a) while the surface viscosities, $\eta_{X P C S}$, are comparable to that in the bulk, $\eta_{\mathrm{XPCS}} \approx \eta_{0}$, Fig. $2 \mathrm{a}$. This result also indicates that the surface dynamics measured by XPCS, and at the corresponding time-scales, encompass those of the center of mass translational dynamics for $T>T_{\mathrm{g}}^{\text {bulk }}+30 \mathrm{~K}[14,22]$. It should be emphasized that only at temperatures close to $T_{\mathrm{g}}$, i.e. at $T_{\mathrm{g}}<T<T_{\mathrm{g}}+20 \mathrm{~K}$, the faster segmental dynamics of the surface layer were manifested within the time $\left(1-10^{3} \mathrm{sec}\right)$ and length scales $\left(10-10^{3} \mathrm{~nm}\right)$ of the XPCS measurements and the surface relaxation times were shorter than that of the corresponding bulk zero shear viscosity .

For 8-arms SPS films with $M_{\mathrm{w}}=25$ and $57 \mathrm{~kg} / \mathrm{mol}$, the HCT describes the $\tau$ versus $q_{\|}$ dependence quite well (Fig. 1b). The data in Fig. $2 \mathrm{~b}$ show the differences between $\eta_{\mathrm{XPCS}}$ and $\eta_{\mathrm{o}}$, (see supplementary information [21] and also Ref [23]), for the SPS-8-25K and SPS-8-57K samples. In order to extract the viscosities of the SPS molecules, their surface tensions were estimated using the work of Archer and co-workers [24,25]. For the slightly entangled SPS-8$57 \mathrm{~K}, \eta_{\mathrm{XPCS}}$ is slightly lower than $\eta_{0}$ whereas for SPS- $8-25 \mathrm{~K} \eta_{\mathrm{XPCS}}$, at the lowest temperature, is smaller than $\eta_{0}$ by an order of magnitude. The value of $\eta_{\text {XPCS }}$ becomes comparable to $\eta_{0}$ for the highest temperature $T \sim T_{\mathrm{g}}+50 \mathrm{~K}$. With regard to SPS-8-10K, the HCT model completely fails to describe the $\tau-\mathrm{vs}-q_{\|}$data throughout the entire temperature ranges. This is shown in Fig. 3, 
where the solid symbols represent the XPCS data and the solid lines were computed with the HCT model (using the values of bulk zero-shear viscosities).

The $q$-dependence of the relaxation times of the SPS- $8-10 \mathrm{~K}$ molecules is instructive with regard to identifying potential differences between the relaxation mechanisms of this polymer and the others. For $T \approx T_{\mathrm{g}}+40 \mathrm{~K}$ and $T_{\mathrm{g}}+50 \mathrm{~K}$ the relaxation times scale as $\tau \sim 1 / q$. Additionally, the relaxation times are significantly longer than the relaxation times in the bulk. These combined observations suggest that the dynamics of the molecules at the free surface are "caged" [26,27]. We will return to this point later in our discussion.

The stretching exponents $\left(\alpha_{\mathrm{XPCS}}\right)$ of the intermediate scattering functions, obtained from the XPCS measurements, shown in Fig. 4. For the linear chain PS samples, $\alpha_{\mathrm{XPCS}}=1$ as expected for the experimental window which captures only translational relaxations [14]. For star-shaped PS samples, $\alpha_{\mathrm{XPCS}}$ is not only dependent on $M_{\mathrm{w}}{ }^{\mathrm{arm}}$ but it is also $T$ dependent, increasing with $T$. A stretched exponent of $\alpha_{\mathrm{XPCS}}<1$ suggests that a distribution, relaxation process occurs at a given temperature. For the highest temperature $T=T_{\mathrm{g}}+50 \mathrm{~K}, \alpha_{\mathrm{XPCS}}=1$ for the SPS-8-25K and SPS-8$10 \mathrm{~K}$, suggesting that the same dynamics processes, collective mass motions, are captured by the XPCS experiment. However, for the SPS-8-57K molecules the values of $\alpha_{\mathrm{XPCS}}$ are much smaller, throughout the entire temperature range, suggesting a broader distribution of relaxation times [28]. With the use of molecular dynamics simulations, we recently showed that due to their architecture and associated monomer packing frustrations, the dynamics of star-shaped polymers would be characterized by broader distributions of relaxation times than their linear chain analogs: dynamics are slower at the vicinity of the branched point (higher monomer density) and become faster towards the arm end (lower monomer density) [15]. The distribution becomes broader as functionality increases and/or when the arm is shorter. So one could ask, what is the 
effect of the structure and dynamical intra-molecular heterogeneity of star polymers in the dynamics measured with XPCS? Our data reveal that the broadening of the XPCS surface dynamics of the SPS-8-57K is not due to intermolecular heterogeneities in the segmental dynamics, as it has been seen in the bulk for star-shaped molecules [15], but rather due to the effect of interfacial interactions on the free surface dynamics. Furthermore, the failure of the HCT to describe the $\tau$ - $q_{\|}$behavior of the SPS- $8-10 \mathrm{~K}$ molecule is associated with the molecular packing of these molecules at the free interface that requires cooperative rearrangements (collective motions) for surface relaxations.

Further insights into these results -the failure of the HCT to describe the $\tau-q_{\|}$behavior of the SPS-8-10K molecule and the trend in the $\alpha_{\mathrm{XPCS}}$ exponent with arm length - may be gleaned from bead-spring coarse-grained molecular dynamics (MD) simulations. In this model, each starshaped polymer molecule is represented by a bead (core) onto which 8 chains, each with $M$ segments of a diameter $\sigma$, is attached. The core particle has a diameter of $0.5 \sigma$. Thin films of thickness $H$ were "constructed" such that one interface was exposed to vacuum and the other was in contact with a solid substrate. Simulations of thin films of star polymers having $M=10$ segments per arm were performed; details of the simulations are described elsewhere [20,29]. Based on previous studies [20,29], we know that there is a stronger positional correlation (concentration profile) of the core particles at the free interface for films having 8-arm star polymers than the linear chain analogues; this implies that star polymers are preferentially located at the free surface compared to the interior of the film, as illustrated in Fig. 5a. In other words, this preferential localization means that the excess number of star molecules at the interface compared to the bulk is expected to enhance the dynamical frustration. This is an 
additional effect associated with the emergence of particle-like behavior and molecular packing with increasing $f$ in the bulk as discussed in a previous study [30].

Based on the foregoing it is evident that the slow surface dynamics determined using XPCS compared to the bulk viscosity for the SPS-8-10K system, not exhibited by linear chain polymers, are associated with the molecular packing of the macromolecules at the free surface due to the preferential localization. The additional findings that $\tau \sim q^{-1}$ suggest evidence that the dynamics of the molecules are caged [26], and is consistent with the slower surface dynamics measured with XPCS, as the relaxation will occur via a slower cooperative process.

In conclusion, we have shown that for temperatures far above $T_{\mathrm{g}}$, the surface dynamics of thin film SPS molecules with $M_{\mathrm{arm}}<M_{\mathrm{e}}$ are slow compared to the bulk. The surface relaxations occur via a slow cooperative process. These are new insights into polymer dynamics contrast to the well-understood behavior of linear-chain polymers where the surface dynamics can be fast compared to the bulk. MD simulations of supported films reveal that the star-shaped molecules exhibit preferential localization at the free surface in contrast to the bulk. Recent oscillatory shear experiments reveal that for bulk systems, the molecules with $f \geq 8$ and $M_{\text {arm }}<M_{e}$ undergo flow via a cooperative mechanism, unlike the conventional arm retraction mechanism characteristic of the long-range dynamics of long armed $\left(M_{\mathrm{arm}}>M_{\mathrm{e}}\right)$ star shaped macromolecules [23]. This new phenomenon -slow surface dynamics- should be general and exhibited by branched molecules whose motions become "caged" due to a tendency toward aggregation or localization at the free surface. These findings have important implications with the regard to the use of polymers for applications that include lubrication, tribology, and adhesion. 


\section{Acknowledgements:}

This research was supported by the National Science Foundation (NSF), Division of Materials Research (DMR-1305749). The use of Advanced Photon Source was supported by the US Department of Energy, Office of Science, Office of Basic Energy Science, under Contract No. DE-AC02-06CH11357.

+ Current address: Materials Science and Engineering Division, National Institute of Standards and Technology, Gaithersburg, Maryland, 20 899, USA

*Authors to whom correspondence should be address: eglynos@iesl.forth.gr, pfgreen@umich.edu, peter.green@NREL.gov.

\section{References}

[1] P. F. Green, E. Glynos, and B. Frieberg, Mrs Communications 5, 423 (2015).

[2] S. Napolitano, S. Capponi, and B. Vanroy, Eur. Phys. J. E 36, 37, 61 (2013).

[3] M. Alcoutlabi and G. B. McKenna, J. Phys.-Condes. Matter 17, R461 (2005).

[4] J. A. Forrest and K. Dalnoki-Veress, Adv. Colloid Interface Sci. 94, 167 (2001).

[5] S. Napolitano, E. Glynos, and N. B. Tito, Reports on Progress in Physics 80, 036602 (2017).

[6] B. Frieberg, E. Glynos, and P. F. Green, Phys. Rev. Lett. 108, 268304 (2012).

[7] M. D. Ediger and J. A. Forrest, Macromolecules 47, 471 (2014).

[8] K. Paeng, S. F. Swallen, and M. D. Ediger, Journal of the American Chemical Society 133, 8444 (2011).

[9] K. Paeng, R. Richert, and M. D. Ediger, Soft Matter 8, 819 (2012).

[10] Z. Fakhraai and J. A. Forrest, Science 319, 600 (2008).

[11] J. L. Harden, H. Pleiner, and P. A. Pincus, J. Chem. Phys. 94, 5208 (1991).

[12] J. Jackle, J. Phys.-Condes. Matter 10, 7121 (1998).

[13] S. K. Sinha, Z. Jiang, and L. B. Lurio, Adv. Mater. 26, 7764 (2014).

[14] Z. Jiang, M. K. Mukhopadhyay, S. Song, S. Narayanan, L. B. Lurio, H. Kim, and S. K. Sinha, Phys. Rev. Lett. 101, 4 (2008).

[15] A. Chremos, E. Glynos, and P. F. Green, J. Chem. Phys. 142, 9, 044901 (2015).

[16] S. F. Wang, Z. Jiang, S. Narayanan, and M. D. Foster, Macromolecules 45, 6210 (2012).

[17] S. F. Wang, S. Yang, J. Lee, B. Akgun, D. T. Wu, and M. D. Foster, Phys. Rev. Lett. 111, 068303 (2013).

[18] B. X. Liu, S. Narayanan, D. T. Wu, and M. D. Foster, Macromolecules 46, 3190 (2013).

[19] E. Glynos, B. Frieberg, H. Oh, M. Liu, D. W. Gidley, and P. F. Green, Phys. Rev. Lett. 106, 128301 (2011).

[20] E. Glynos, B. Frieberg, A. Chremos, G. Sakellariou, D. W. Gidley, and P. F. Green, Macromolecules 48, 2305 (2015).

[21] See Supplemental Material at [url] for specific details, which includes Ref. [31-33]

[22] H. Kim, A. Ruhm, L. B. Lurio, J. K. Basu, J. Lal, D. Lumma, S. G. J. Mochrie, and S. K. Sinha, Phys. Rev. Lett. 90, 4, 068302 (2003). 
[23] K. J. Johnson, E. Glynos, G. Sakellariou, and P. Green, Macromolecules 49, 5669 (2016).

[24] V. S. Minnikanti and L. A. Archer, Macromolecules 39, 7718 (2006).

[25] Z. Y. Qian, V. S. Minnikanti, B. B. Sauer, G. T. Dee, and L. A. Archer, Macromolecules 41, 5007 (2008).

[26] S. Srivastava, P. Agarwal, R. Mangal, D. L. Koch, S. Narayanan, and L. A. Archer, ACS Macro Lett. 4, 1149 (2015).

[27] C. Caronna, Y. Chushkin, A. Madsen, and A. Cupane, Phys. Rev. Lett. 100, 4, 055702 (2008).

[28] M. L. Ruegg, A. J. Patel, S. Narayanan, A. R. Sandy, S. G. J. Mochrie, H. Watanabe, and N. P. Balsara, Macromolecules 39, 8822 (2006).

[29] E. Glynos, A. Chremos, B. Frieberg, G. Sakellariou, and P. F. Green, Macromolecules 47, 1137 (2014).

[30] A. Chremos and J. F. Douglas, J. Chem. Phys. 143, 111104 (2015).

[31] N. Hadjichristidis, H. latrou, S. Pispas, and M. Pitsikalis, J. Polym. Sci. Pol. Chem. 38, 3211 (2000)

[32] D. Uhrig and J. W. Mays, J. Polym. Sci. Pol. Chem. 43, 6179 (2005)

[33] J. Roovers, L. L. Zhou, P. M. Toporowski, M. Vanderzwan, H. Iatrou, and N. Hadjichristidis, Macromolecules 26, 4324 (1993) 


\section{Figures:}
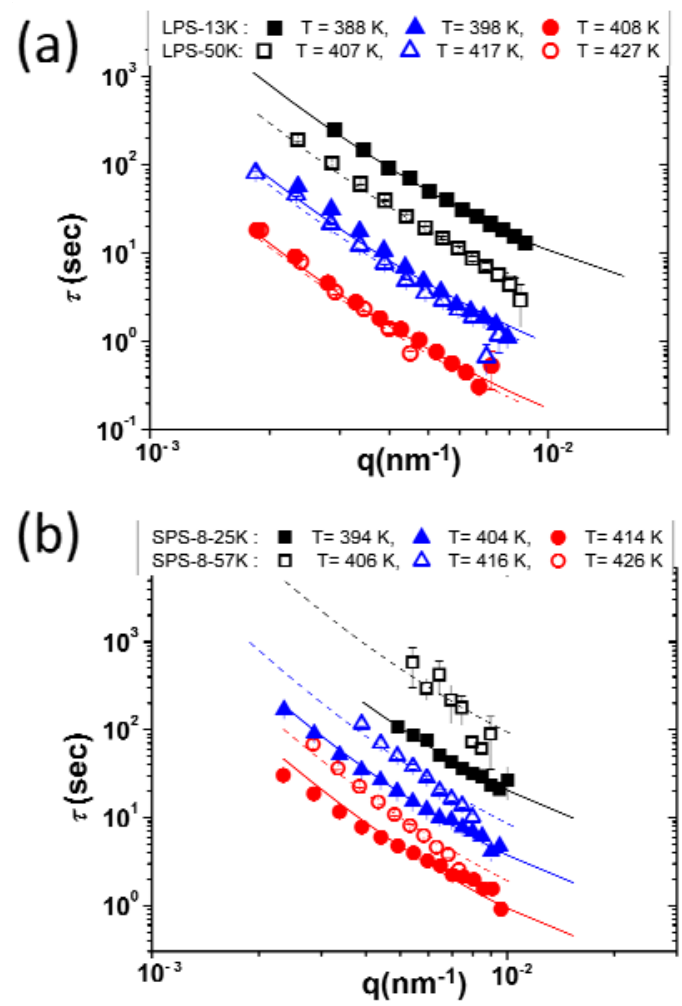

Figure 1: Relaxation times as a function of wave function for various PS films at different temperatures, $T$, for: (a) LPS-13K (solid symbols) and LPS 50K (open symbols); (b) SPS-8-25K (solid symbols) SPS-8-57K (open symbols). The curves represent the least-squared fits of data to the HCT. 

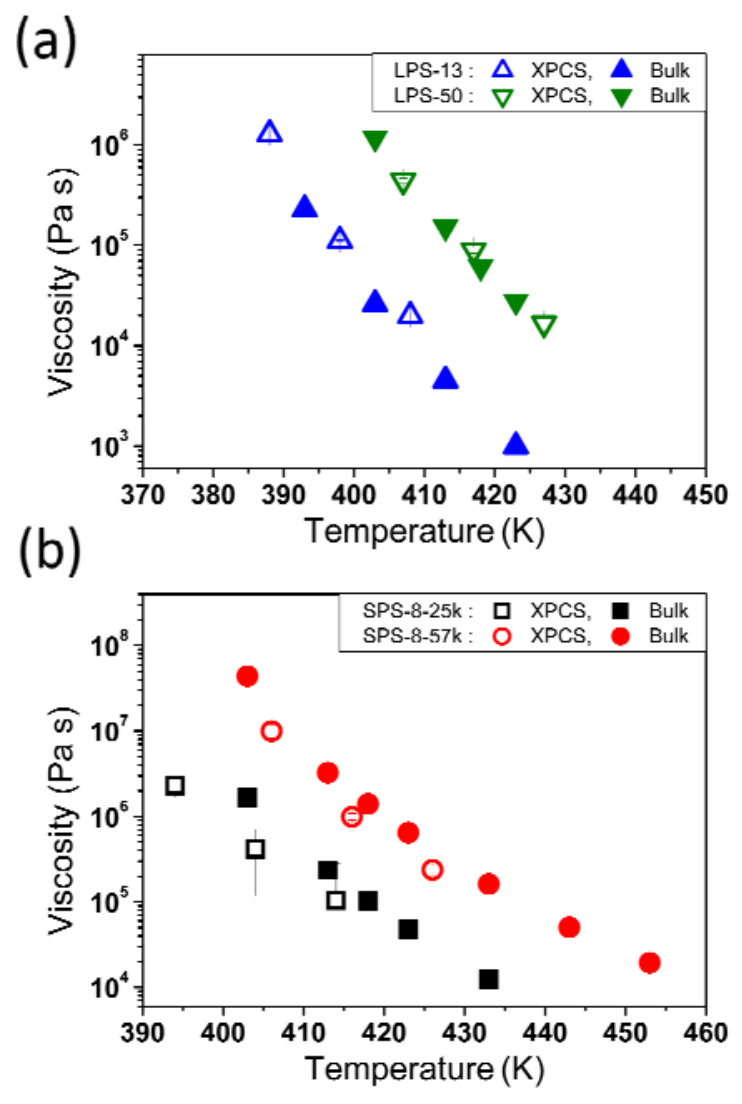

Figure 2: A comparison of viscosities of films determined using XPCS data (filled symbols) and bulk viscosities measured by rheometry (open symbols) as a function of $\Delta T_{\mathrm{g}}$ for (a) LPS-13K (blue symbols) and LSP-50K (green symbols) and (b) SPS-8-25K (black symbols) and SPS-857K (red symbols). 


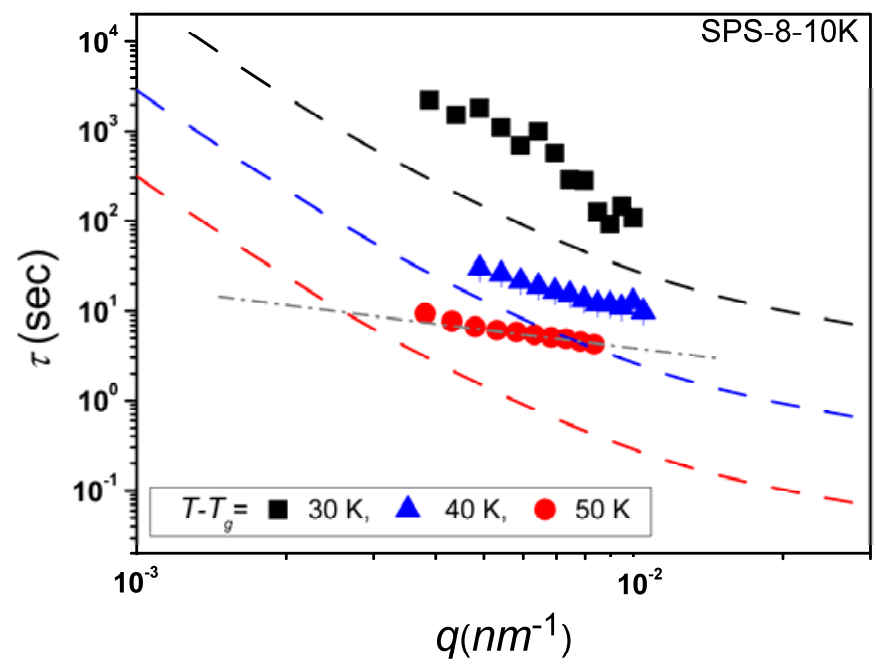

Figure 3: XPCS surface relaxation time for the SPS-8-10K measured at different temperatures. The solid curves represent the estimated surface relaxation times based on HCT using the zeroshear viscosity. The dashed line indicates the $\tau \sim q^{-1}$.

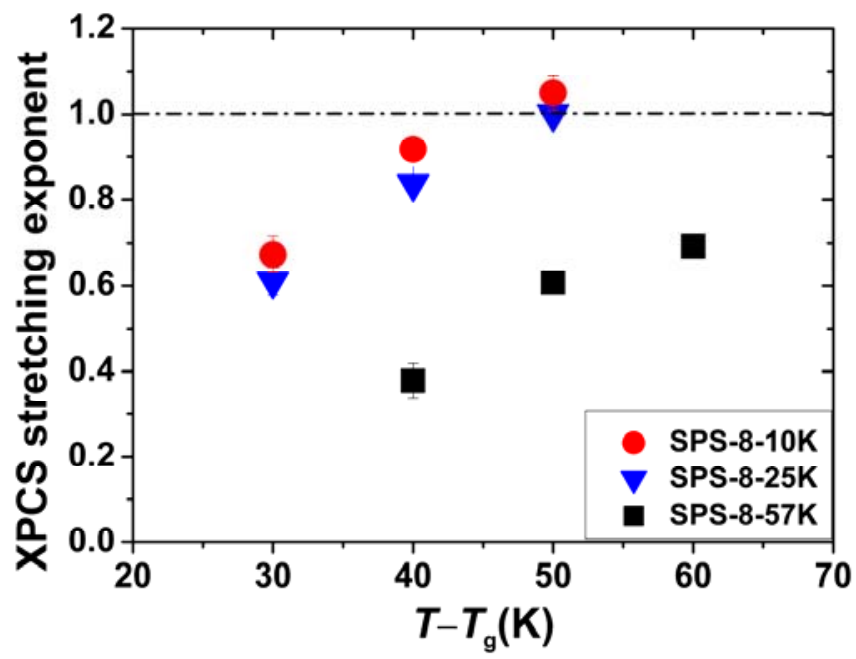

Figure 4: XPCS stretching exponents as a function of $\Delta T_{\mathrm{g}}$ for 8-arm films with $M_{\mathrm{w}}{ }^{\mathrm{arm}}=10 \mathrm{~kg}$ / mol (solid red symbols), $25 \mathrm{~kg} / \mathrm{mol}$ (solid blue triangle), and $57 \mathrm{~kg} / \mathrm{mol}$ (solid black squares). 


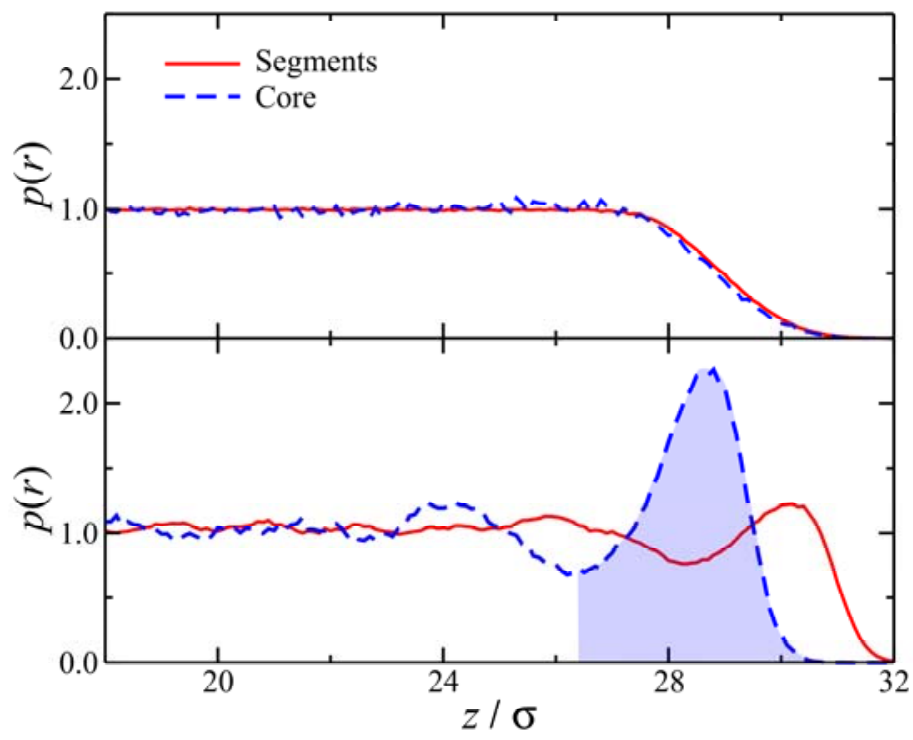

Figure 5: Segmental and core particle concentration profiles for stars having (top) $f=2$ arms (i.e linear chains) and (bottom) $f=8$-arms and $M=10$ segments per arm. The highlighted region corresponds to the star polymer interfacial layer at the free surface and reflects the preferential localization of stars at the free interface. 